\title{
Characterisation of PHEMT intermodulation behaviour for highly linear MMIC power amplifier design
}

\author{
M. Pagani, D. Argento, M. Bignamini, I. De Francesco, G. Favre, A. Meazza, A. Mornata, and \\ F. Palomba \\ ERICSSON LAB ITALY \\ Via Cadorna, 73 - 20090 - Vimodrone (MI) - Italy
}

\begin{abstract}
In this paper the intermodulation distortion (IMD) behaviour of a 0.25 power PHEMT is investigated under several device operating conditions. An extensive experimental device characterisation, together with numerical simulations using suitable non-linear transistor models is carried out. Experimental data were compared with different transistor models in order to understand the effect of the various cell parameters on the device IMD $\mathrm{I}_{3}$ response. The data collected were applied in the design of a highly linear power amplifier family, covering different frequency ranges.
\end{abstract}

\section{INTRODUCTION}

Highly linear power amplifiers are key components for microwave radios using digital modulation schemes with high spectral efficiency. Improving trade-offs between linearity and power added efficiency (PAE) is extremely important to this kind of application so that several studies have been recently presented addressing this issue. We also proposed an approach for custom MMIC design where a trade-off between linearity and PAE was considered from the beginning of the design activity (Bignamini et al. (1)). For this purpose a successful design will necessarily be based on a suitable non linear model, able to predict both gain and $\mathrm{IMD}_{3}$ distortion with a certain degree of precision, or on a sufficiently large amount of experimental data.

In this paper we investigate the non-linear behaviour of a 0.25 power PHEMT as a function of many operational parameters, as frequency, source and load impedance. The data collected are analysed in many different frameworks, first adopting extremely simplified models, then using more accurate models, in order to understand the origin of the observed behaviour.

\section{EXPERIMENTAL SET UP}

A block diagram of the experimental set up, is shown in fig. 1. Two RF signals are independently generated and amplified before being combined at the input of DUT. The PHEMT is biased by means of bias -tees (omitted in the picture). The source and load impedance are tuned by means of two passive tuners and measured with a HP8510 network analyzer. In order to extend the

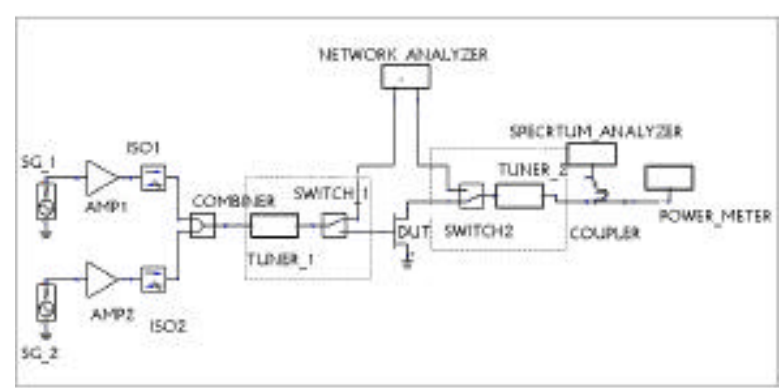

Figure 1: Experimental set up

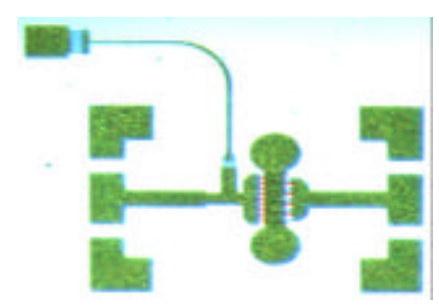

Figure 2:Test device with the pre-matching network

tunability range of input cell terminations to the low resistance region, a pre-matching network was designed (see fig. 2).

The input and output network are connected to the DUT by means of two switches. A $10 \mathrm{~dB}$ directional coupler at the output allows the simultaneous measurement of output power and $\mathrm{IMD}_{3}$ distortion. 


\section{RESULTS}

In this paper we focus our attention on a set of measurements performed on a $600 \mu \mathrm{m}$ PHEMT @ 13 $\mathrm{GHz}$, biased with $\mathrm{V}_{\mathrm{d}}=6.5 \mathrm{~V}$ and $\mathrm{I}_{\mathrm{d}}=60 \mathrm{~mA}$. In fig. 3 are shown IMD measurements performed with the load impedance fixed at its optimum value, previously determined with a $50 \mathrm{Ohm}$ input termination $\left(Z_{\text {out }}=(27+j 32 \Omega)\right.$, for different values of the resistive part of the source impedance.

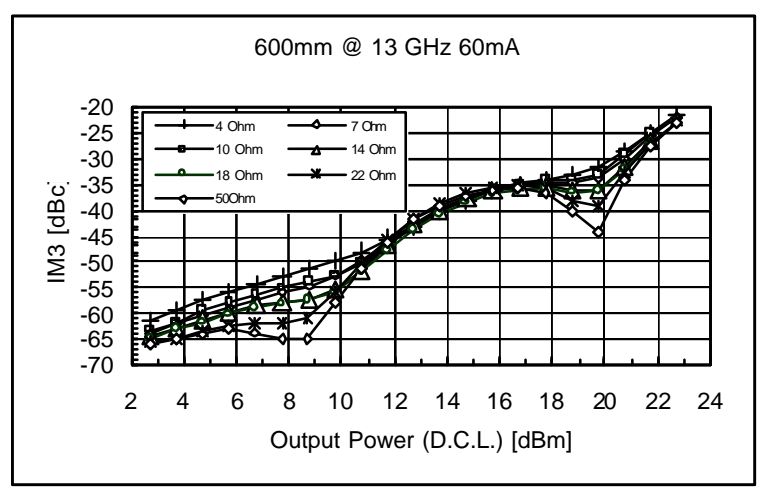

Figure 3: $I M D_{3}$ distortion as a function of output power for different source impedances

The reactive part was also kept constant and equal to zero. Although at a very low power level $\mathrm{IMD}_{3}$ is unaffected by the source termination, increasing the power the presence of two "spots" in $\mathrm{IMD}_{3}$ distortion can be observed.

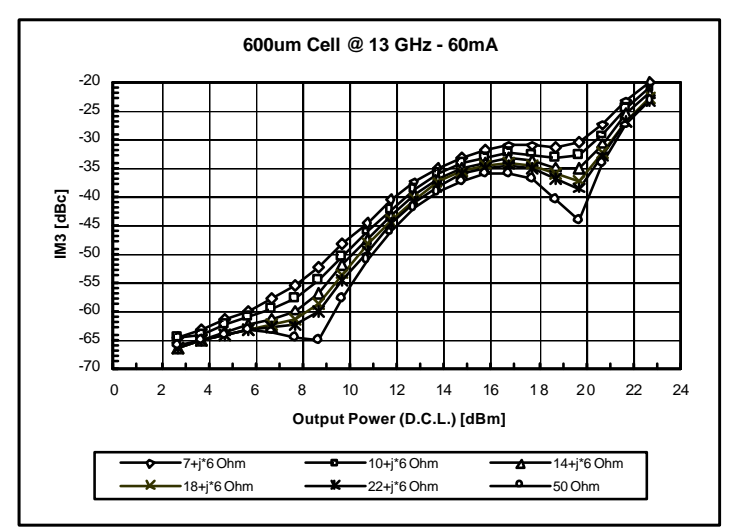

Figure 4: $\mathrm{IMD}_{3}$ as a function of output power for different source impedances with a non zero reactive component.
The dependence of $\mathrm{IMD}_{3}$ by source impedance disappears in a moderately high power level range.

In figure 4 are shown measurements performed introducing a reactive part in the source impedance. The behaviour is similar, but in this case changes in $\mathrm{IMD}_{3}$ distortion with the input termination can be observed in the whole high power range.

\section{DISCUSSION}

To understand the origin of the observed behaviour, and in particular the parameters that influence the $\mathrm{IMD}_{3}$ spots, we analysed the experimental data in different frameworks. The simplest model we considered is the widely accepted equivalent circuit shown in fig. 6 . We adopted the following strong simplifications:

- The dependence of all parameters by $V_{d}$ was not considered.

- The only non linear components we considered were the gate-source capacitance $\left(\mathrm{C}_{\mathrm{gs}}\right)$ and the drain current generator $\left(\mathrm{G}_{\mathrm{m}}\right)$, as non-linear function of $V_{\mathrm{gs}}$.

To extract the model parameters we proceeded as follows. We measured the cell $\mathrm{S}$ parameters at a moderately low frequency $(900 \mathrm{MHz}$ ) as a function of the gate bias voltage $V_{g s}$. In this frequency range the input and output parasitic capacitances can be neglected. From the $\mathrm{S}$ parameters we calculated the $\mathrm{Z}$ parameters. Then we evaluated the $Z$ parameters of the intrinsic model de-embedding the parasitic impedance at the cell terminals:

$$
Z^{\prime}=Z-Z_{e}
$$

with

$$
\mathbf{Z}_{\mathbf{e}}=\left[\begin{array}{cc}
Z_{s}+Z_{g} & Z_{s} \\
Z_{s} & Z_{s}+Z_{d}
\end{array}\right]
$$

The value of $Z_{g}, Z_{s}$, and $Z_{d}$, were given by the foundry. From $Z^{\prime}$ we calculated the intrinsic admittance matrix $Y^{\prime}$ and then the required parameters:

$$
\begin{gathered}
C_{g d}=-\frac{\operatorname{Im}\left(Y_{12}^{\prime}\right)}{\omega} \\
R_{i}=\operatorname{Re}\left(\frac{1}{Y_{11}^{\prime}-j \omega C_{g d}}\right)
\end{gathered}
$$




$$
\begin{gathered}
C_{g s}=-\frac{1}{\omega \operatorname{Im}\left(\frac{1}{Y_{11}^{\prime}-j \omega C_{g d}}\right)} \\
R_{d s}=\frac{1}{\operatorname{Re}\left(Y_{22}^{\prime}-j \omega C_{g d}\right)} \\
C_{d s}=\frac{\operatorname{Im}\left(Y_{22}^{\prime}-j \omega C_{g d}\right)}{\omega} \\
G_{m}=\left|\left(Y_{21}^{\prime}+j \omega C_{g d}\right)\left(1+j \omega C_{g s} R_{i}\right)\right| \\
\tau=-\frac{\left.\angle\left(Y_{21}^{\prime}+j \omega C_{g d}\right)\left(1+j \omega C_{g s} R_{i}\right)\right]}{\omega}
\end{gathered}
$$

As a further approximation, $\mathrm{C}_{\mathrm{gs}}$ and $\mathrm{G}_{\mathrm{m}}$ behaviour was approximated in a small region around the bias point with a $5^{\text {th }}$ order polynomial function.

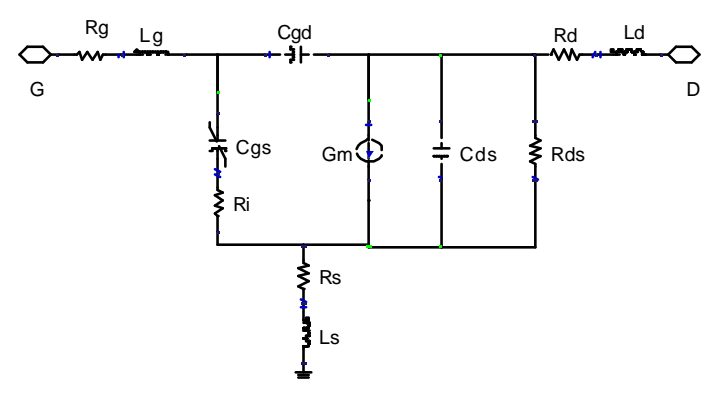

Figure 5: PHEMT equivalent circuit

In order to separately analyse the $\mathrm{C}_{\mathrm{gs}}$ and $\mathrm{G}_{\mathrm{m}}$ contribution to $\mathrm{IMD}_{3}$ distortion, we first simulated the circuit with a non-linear $\mathrm{C}_{\mathrm{gs}}$ and a linear $\mathrm{G}_{\mathrm{f}}$. Then we simulated a circuit with a linear $C_{g s}$ and a non-linear $G_{m}$. As final step we introduced both $\mathrm{C}_{\mathrm{gs}}$ and $\mathrm{G}_{\mathrm{n}}$ non-linearities. For each of this case was performed a source pull. Some of the results obtained are shown in fig. 6 and 7 and can be summarised as follows:

- The high power spot can be easily reproduced simply considering the $\mathrm{G}_{\mathrm{m}}$ non-linearities up to the $5^{\text {th }}$ order and is the effect of the 180 degree out of phase combination of $\mathrm{IMD}_{3}$ coming from the $3^{\text {rd }}$ order term with the one due to the $5^{\text {th }}$ order term.

- Even if the framework is extremely simplified, the model predicts a remarkable influence of source termination on IMD3. This effect, present in our measurements (fig. 3 and 4), is widely discussed in literature (J. A. Garcia et al. (2), Kwang-Ho Ahn et al. (3)).

- The introduction of non-linearities in the $\mathrm{C}_{\mathrm{g}}$ decrease $\mathrm{IMD}_{3}$ over the entire power range considered (fig. 6). The Intermodulation distortion decrease depends on the phase difference between the $\mathrm{IMD}_{3}$ due to $\mathrm{C}_{\mathrm{gs}}$ and the one due to $\mathrm{G}_{\mathrm{m}}$.

The low power spot cannot be explained in this simplified scheme because the phase difference of $G_{n}$ and $\mathrm{C}_{\mathrm{g}}$ non-linearities is almost independent by the power level. For this reason the two non-linearities will combine always in the same way, increasing or decreasing the overall $\mathrm{IMD}_{3}$. distortion, having the same effect over the whole power range.

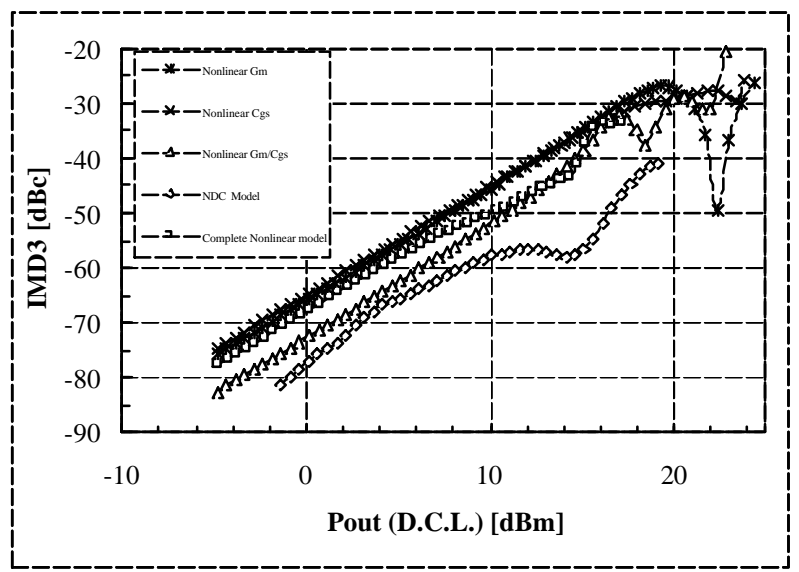

Figure 6: Comparison of different simulations performed with various non linear models with $Z_{\text {in }}=22 \Omega$ and $Z_{\text {out }}=(27+j 32) \Omega$

To complete the analysis we compared our experimental data with more accurate models. A spot-like behaviour can be observed using the same equivalent circuit of fig. 5 , but introducing other non linear elements (as $R_{\mathrm{ds}}$ and $\mathrm{C}_{\mathrm{gd}}$ ). A better agreement can be obtained using a more sophisticated model taking into account other relevants effect $\left(\mathrm{V}_{\mathrm{d}}\right.$ dependence, memory effects, etc. $)$. For example a low power spot can be observed in a wide range of load and source impedance with the Nonlinear Discrete Convolution (NDC) Model discussed in Filicori 
et al. (4) and Costantini et al. (5). This model takes into account the short time memory effect of the device and predicts its non linear behaviour on the basis of the device small signal behaviour.

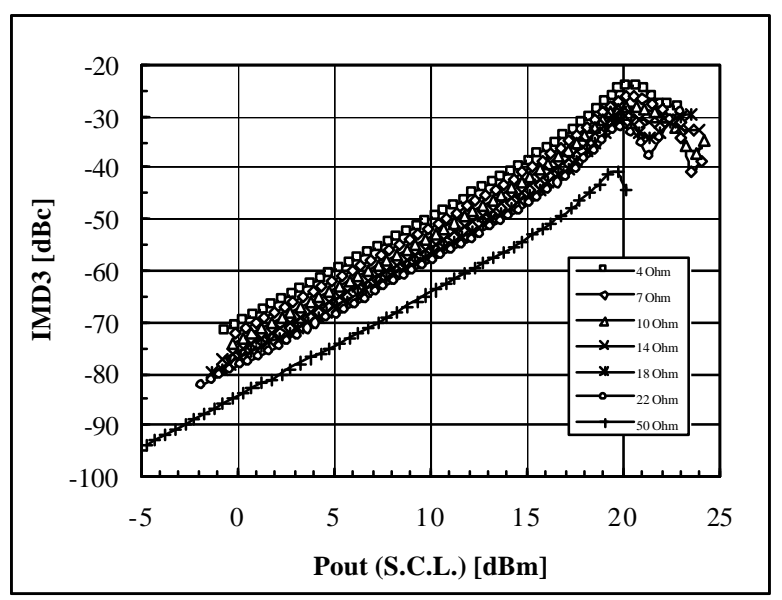

Figure 7: Source pull with the simplified nonlinear model

However, the complexity and the generality of the NDC approach doesn't allow an immediate interpretation of the spot presence in terms of the device parameters.

\section{CONCLUSIONS}

As a step of a complete development procedure set up to design highly linear power amplifiers, a characterisation of the third order intermodulation distortion behaviour of a 0.25 power PHEMT technology was investigated. An extensive experimental device characterisation, together with several numerical simulations, were performed. Starting from very simplified models we discussed separately the effects of the non linear gate-source capacitance and the non linear drain current generator, in order to understand the observed device behaviour. Some of the main observed features can be easily explained even in a very simplified framework. The accurate prediction of the device behaviour and other peculiar effects, as the low power spot, can be addressed only using more complete non-linear models. An example can be the Non-Linear Discrete Convolution Model described in ref. (4), (5).

\section{REFERENCES}

(1) M. Bignamini, G. Favre, A. Meazza, M. Pagani, F. Palomba, and G. Sivverini, Efficient Development of Highly Linear MMIC Power Amplifiers, Proc. GAAS 2001,. ExCel,. London, pp. 693-696, 24-25 September 2001.

J. A. Garcia, A. Mediavilla, J. C. Pedro, N. B. Carvalho, A. Tazon, and J. L. Garcia, Characterizing the Gate to Source Nonlinear Capacitor Role on FET IMD Performance, IEEE Trans. Microwave TheoryTech., Vol. 46 pp. 2344-2347, November 1998.

(3)

Kwang-Ho Ahn, Yoon-Ha Jeong, and Soong Hak Lee, Effect of Source and Load Impedance on the Intermodulation Products of GaAs FETs, MTT-S International 2000, Vol.1 pp. 469-472.

(4)

F. Filicori, G. Vannini, A. Santarelli, A FiniteMemory Nonlinear Modelfor Microwave Electron Device, Proc. $27^{\text {th }}$ EuMC, September 1997.

(5)

A. Costantini, R. P. Paganelli, P. A. Traverso, D. Argento, G. Favre, M. Pagani, A. Santarelli, G. Vannini, and F. Filicori, Accurate Prediction of PHEMT Intermodulation Distortion Using the Nonlinear Discrete Convolution Model, MTT-S International 2002, Vol. 2, pp. 857-860, Washington, June 2002. 